Acta Crystallographica Section E

Structure Reports

Online

ISSN 1600-5368

\section{Christopher Bresner, Simon Aldridge,* Ian A. Fallis and Li-Ling Ooi}

School of Chemistry, Cardiff University, PO Box 912, Park Place, Cardiff CF10 3TB, Wales

Correspondence e-mail: aldridges@cf.ac.uk

\section{Key indicators}

Single-crystal X-ray study

$T=180 \mathrm{~K}$

Mean $\sigma(\mathrm{C}-\mathrm{C})=0.006 \AA$

$R$ factor $=0.054$

$w R$ factor $=0.105$

Data-to-parameter ratio $=16.5$

For details of how these key indicators were automatically derived from the article, see http://journals.iucr.org/e.

\title{
Hydrogen-bonding motifs in the solid-state structure of ferroceneboronic acid
}

At $180 \mathrm{~K}$, the crystal structure of ferroceneboronic acid, $\left[\mathrm{Fe}\left(\mathrm{C}_{5} \mathrm{H}_{5}\right)\left(\mathrm{C}_{5} \mathrm{H}_{6} \mathrm{BO}_{2}\right)\right]$, consists of centrosymmetric $\left[\mathrm{FcB}(\mathrm{OH})_{2}\right]_{2}$ dimers $\left[\mathrm{Fc}\right.$ is $\left.\left(\eta^{5}-\mathrm{C}_{5} \mathrm{H}_{5}\right) \mathrm{Fe}\left(\eta^{5}-\mathrm{C}_{5} \mathrm{H}_{4}\right)\right]$, formed by a pair of complementary $\mathrm{O}-\mathrm{H} \cdots \mathrm{O}$ hydrogen-bonding interactions $[\mathrm{O} \cdots \mathrm{H} \quad 1.97 \AA$ and $\mathrm{O} \cdots \mathrm{O} \quad 2.806(3) \AA]$. The remaining two O-bound $\mathrm{H}$ atoms per $\left[\mathrm{FcB}(\mathrm{OH})_{2}\right]_{2}$ moiety serve to link the dimeric units to adjacent dimers in a crisscross fashion, very similar to that between hydrogen-bonded chains in solid $\mathrm{Fe}\left[\eta^{5}-\mathrm{C}_{5} \mathrm{H}_{4} \mathrm{~B}(\mathrm{OH})_{2}\right]_{2}$.

\section{Comment}

The title compound, ferroceneboronic acid, $\mathrm{FcB}(\mathrm{OH})_{2}[\mathrm{Fc}$ is $\left.\left(\eta^{5}-\mathrm{C}_{5} \mathrm{H}_{5}\right) \mathrm{Fe}\left(\eta^{5}-\mathrm{C}_{5} \mathrm{H}_{4}\right)\right]$, has been known since the late $1950 \mathrm{~s}$, having been initially synthesized by Nesmeyanov et al. (1959) by the reaction of lithioferrocene with $(\mathrm{BuO})_{3} \mathrm{~B}$, and subsequent hydrolytic work-up. A number of alternative syntheses have been reported in the interim (Shechter \& Helling, 1961; McVey et al., 1967), and continued interest in this compound and related derivatives is in part due to their implication in Suzuki-type coupling reactions (Hua et al., 2001) and in anion and neutral molecule sensing (Dusemund et al., 1995; Ori \& Shinkai, 1995). Although the solid-state structures of a number of related compounds, including the diboronic acid $\mathrm{Fe}\left[\eta^{5}-\mathrm{C}_{5} \mathrm{H}_{4} \mathrm{~B}(\mathrm{OH})_{2}\right]_{2}$ (Braga et al., 2003), the cyclic boronic anhydride $(\mathrm{FcBO})_{3}$ (Bats et al., 2002), boronic esters (such as $\mathrm{FcBO}_{2} \mathrm{C}_{6} \mathrm{H}_{4}-1,2$; Aldridge \& Bresner, 2003; Aldridge, Bresner $\&$ Fallis, 2004) and [5]trovacenylboronic acid, $\left(\eta^{7}-\mathrm{C}_{7} \mathrm{H}_{7}\right) \mathrm{V}\left[\eta^{5}-\right.$ $\mathrm{C}_{5} \mathrm{H}_{4} \mathrm{~B}(\mathrm{OH})_{2}$ ] (Elschenbroich et al., 2004), have been determined, to our knowledge there have been no reports to date concerning the crystal structure of $\mathrm{FcB}(\mathrm{OH})_{2}$. The title compound, (I), was isolated in this instance as the main organometallic product from the aerobic hydrolysis of $\mathrm{FcB}\left(\mathrm{OCH}_{2} \mathrm{CH}_{2}\right)_{2} \mathrm{~S}$ in a mixed toluene-hexane solvent (Aldridge et al., 2004).

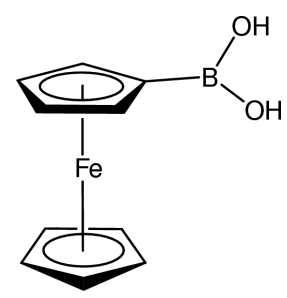

(I)

The structural parameters relating to the individual $\mathrm{FcB}(\mathrm{OH})_{2}$ units of $(\mathrm{I})$ are unremarkable, with the geometries at the $\mathrm{Fe}$ and $\mathrm{B}$ centres mirroring those found in related compounds (Bats et al., 2002; Braga et al., 2003; Aldridge et al.,
Received 27 February 2004 Accepted 15 March 2004 Online 24 March 2004
C 2004 International Union of Crystallography Printed in Great Britain - all rights reserved 


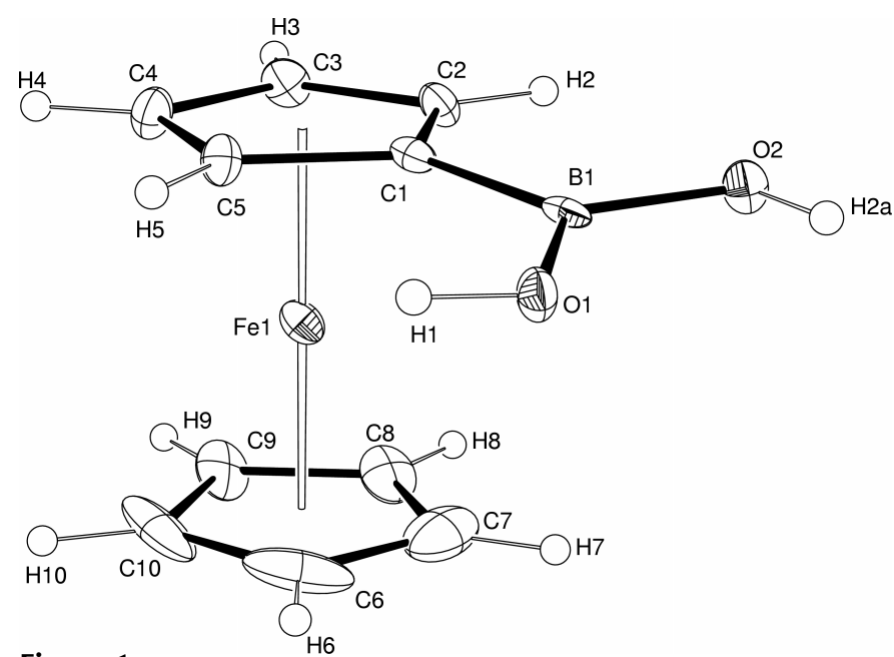

Figure 1

A view of the molecular structure of (I), showing the atom-labelling scheme. Displacement ellipsoids are drawn at the $30 \%$ probability level and $\mathrm{H}$ atoms are represented by spheres of arbitrary size.

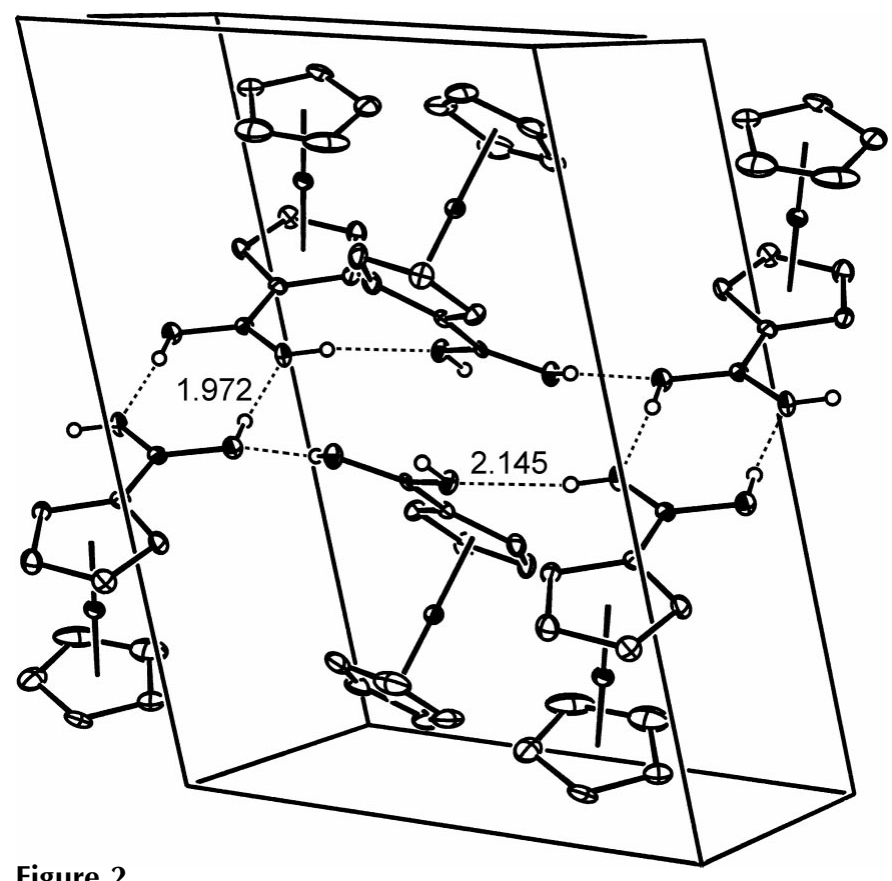

A packing diagram, showing the hydrogen-bonding motifs both within and between the dimeric $\left[\mathrm{FcB}(\mathrm{OH})_{2}\right]_{2}$ units in solid (I). Distances are in A.

2004). In particular, the small degree of bending of the boronic acid moiety out of the plane of the cyclopentadienyl ligand $\left[\mathrm{Cp}\right.$ centroid-C1-B1 $\left.176.4(2)^{\circ}\right]$ mirrors that observed in related ferrocenes containing weakly Lewis acidic boryl $\left(\mathrm{B} X_{2}\right)$ substituents [e.g. $178.5(3)^{\circ}$ for $\mathrm{FcBO}_{2} \mathrm{C}_{2} \mathrm{H}_{2} \mathrm{Ph}_{2}$; Aldridge et al., 2004], but contrasts with that found in the much more electron-deficient $\mathrm{FcBBr}_{2}$ ( $\mathrm{ca} 162^{\circ}$ for both crystallographically independent molecules; Appel et al., 1996; Aldridge \& Bresner, 2003).

In the solid state, the molecular units of (I) aggregate into centrosymmetric dimers, $\left[\mathrm{FcB}(\mathrm{OH})_{2}\right]_{2}$, formed by a pair of complementary $\mathrm{O}-\mathrm{H} \cdots \mathrm{O}$ hydrogen-bonding interactions characterized by $\mathrm{O} \cdots \mathrm{H}$ distances of $1.97 \AA$ and $\mathrm{O}-\mathrm{H} \cdots \mathrm{O}$ angles of $172^{\circ}$. The eight-membered ring thus formed is very similar to that seen in the crystal structures of $\mathrm{Fe}\left[\eta^{5}\right.$ $\left.\mathrm{C}_{5} \mathrm{H}_{4} \mathrm{~B}(\mathrm{OH})_{2}\right]_{2}$ and $\mathrm{PhB}(\mathrm{OH})_{2}$ (Braga et al., 2003; Rettig \& Trotter, 1977). Indeed, the O ‥ O separations between the two components of the dimer [2.806 (3) $\AA$ ] are essentially identical to those found in $\mathrm{Fe}\left[\eta^{5}-\mathrm{C}_{5} \mathrm{H}_{4} \mathrm{~B}(\mathrm{OH})_{2}\right]_{2} \quad[2.81(1) \AA]$. The remaining two O-bound $\mathrm{H}$ atoms per $\left[\mathrm{FcB}(\mathrm{OH})_{2}\right]_{2}$ moiety serve to link the dimeric units to adjacent dimers in a crisscross fashion, very similar to the linking between hydrogenbonded chains in solid $\mathrm{Fe}\left[\eta^{5}-\mathrm{C}_{5} \mathrm{H}_{4} \mathrm{~B}(\mathrm{OH})_{2}\right]_{2}$ (Braga et al., 2003). The 16-membered ring thus formed (Fig. 2) incorporates two $\left[\mathrm{FcB}(\mathrm{OH})_{2}\right]_{2}$ dimers from within the same layer, bridged by two $\mathrm{FcB}(\mathrm{OH})_{2}$ moieties from different dimeric units of the intervening stack. The $\mathrm{O} \cdots \mathrm{H}$ and $\mathrm{O} \cdots \mathrm{O}$ distances [2.14 and 2.930 (3) $\AA$, respectively] are somewhat longer than those found within each dimeric unit, but are consistent with the $\mathrm{O} \cdots \mathrm{O}$ distance found for the very similar structural motif present in $\mathrm{Fe}\left[\eta^{5}-\mathrm{C}_{5} \mathrm{H}_{4} \mathrm{~B}(\mathrm{OH})_{2}\right]_{2}$ [O . . O 2.89 (1) $\AA$ ]

\section{Experimental}

The title compound was isolated as the main organometallic product from the aerobic hydrolysis of $\mathrm{FcB}\left(\mathrm{OCH}_{2} \mathrm{CH}_{2}\right)_{2} \mathrm{~S}$ (Aldridge et al., 2004). Attempted recrystallization of $\mathrm{FcB}\left(\mathrm{OCH}_{2} \mathrm{CH}_{2}\right)_{2} \mathrm{~S}$ in air by hexane diffusion into a toluene solution led to the isolation of $\mathrm{FcB}(\mathrm{OH})_{2}$, (I), as single yellow-orange crystals suitable for X-ray diffraction. Spectroscopic data obtained $\left({ }^{11} \mathrm{~B},{ }^{1} \mathrm{H}\right.$ and ${ }^{13} \mathrm{C} \mathrm{NMR}$, and mass spectrometry) were in agreement with those reported previously (Shechter \& Helling, 1961; McVey et al., 1967).

Crystal data

$\left[\mathrm{Fe}\left(\mathrm{C}_{5} \mathrm{H}_{5}\right)\left(\mathrm{C}_{5} \mathrm{H}_{6} \mathrm{BO}_{2}\right)\right]$
$M_{r}=229.85$
Monoclinic, $P 2_{1} / a$
$a=10.0680(7) \AA$
$b=7.0080(5) \AA$
$c=14.0300(13) \AA$
$\beta=106.320(3)^{\circ}$
$V=950.02(13) \AA^{3}$
$Z=4$

Data collection

Nonius KappaCCD area-detector diffractometer

$\varphi$ scans

Absorption correction: multi-scan (SORTAV; Blessing, 1995)

$T_{\min }=0.824, T_{\max }=0.955$ 6820 measured reflections

\section{Refinement}

Refinement on $F^{2}$

$R\left[F^{2}>2 \sigma\left(F^{2}\right)\right]=0.055$

$w R\left(F^{2}\right)=0.105$

$S=1.03$

2130 reflections

129 parameters

$\mathrm{H}$-atom parameters constrained

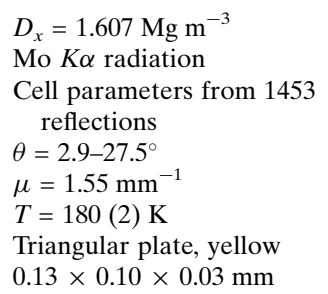

2130 independent reflections 1398 reflections with $I>2 \sigma(I)$ $R_{\text {int }}=0.092$

$\theta_{\max }=27.4^{\circ}$

$h=-12 \rightarrow 12$

$k=-9 \rightarrow 9$

$l=-18 \rightarrow 18$ 
Table 1

Selected geometric parameters $\left(\AA{ }^{\circ},{ }^{\circ}\right.$.

\begin{tabular}{llll}
\hline $\mathrm{Fe} 1-\mathrm{C} 6$ & $2.020(4)$ & $\mathrm{Fe} 1-\mathrm{C} 1$ & $2.054(3)$ \\
$\mathrm{Fe} 1-\mathrm{C} 10$ & $2.032(4)$ & $\mathrm{Fe} 1-\mathrm{C} 8$ & $2.056(4)$ \\
$\mathrm{Fe} 1-\mathrm{C} 7$ & $2.039(4)$ & $\mathrm{C} 1-\mathrm{B} 1$ & $1.551(5)$ \\
$\mathrm{Fe} 1-\mathrm{C} 5$ & $2.043(3)$ & $\mathrm{B} 1-\mathrm{O} 2$ & $1.375(4)$ \\
$\mathrm{Fe} 1-\mathrm{C} 4$ & $2.047(4)$ & $\mathrm{B} 1-\mathrm{O} 1$ & $1.376(4)$ \\
$\mathrm{Fe} 1-\mathrm{C} 3$ & $2.048(3)$ & $\mathrm{O} 1-\mathrm{H} 1$ & 0.84 \\
$\mathrm{Fe} 1-\mathrm{C} 2$ & $2.049(3)$ & $\mathrm{O} 2-\mathrm{H} 2 A$ & 0.84 \\
$\mathrm{Fe} 1-\mathrm{C} 9$ & $2.053(3)$ & & \\
$\mathrm{O} 2-\mathrm{B} 1-\mathrm{O} 1$ & $118.0(3)$ & $\mathrm{O} 1-\mathrm{B} 1-\mathrm{C} 1$ & $123.9(3)$ \\
$\mathrm{O} 2-\mathrm{B} 1-\mathrm{C} 1$ & $118.1(3)$ & & \\
\hline
\end{tabular}

Table 2

Hydrogen-bonding geometry $\left(\AA{ }^{\circ}\right)$.

\begin{tabular}{lllll}
\hline$D-\mathrm{H} \cdots A$ & $D-\mathrm{H}$ & $\mathrm{H} \cdots A$ & $D \cdots A$ & $D-\mathrm{H} \cdots A$ \\
\hline $\mathrm{O} 1-\mathrm{H} 1 \cdots \mathrm{O} 2^{\mathrm{i}}$ & 0.84 & 2.14 & $2.930(3)$ & 156 \\
$\mathrm{O} 2-\mathrm{H} 2 A \cdots \mathrm{O} 1^{\mathrm{ii}}$ & 0.84 & 1.97 & $2.806(3)$ & 172 \\
\hline
\end{tabular}

Symmetry codes: (i) $\frac{1}{2}+x,-\frac{1}{2}-y, z$; (ii) $-x,-1-y,-1-z$.

Aromatic $\mathrm{H}$ atoms were constrained as riding atoms, with $\mathrm{C}-\mathrm{H}$ distances of $0.95 \AA$. Hydroxyl $\mathrm{H}$ atoms were located in a difference Fourier map and refined as a rigid rotor, with $\mathrm{O}-\mathrm{H}$ distances of $0.84 \AA$. The $U_{\text {iso }}(\mathrm{H})$ values were fixed at 1.2 times $U_{\text {eq }}(\mathrm{C})$ or 1.5 times $U_{\text {eq }}(\mathrm{O})$.

Data collection: COLLECT (Nonius, 2000); cell refinement: $H K L$ SCALEPACK (Otwinowski \& Minor, 1997); data reduction: $H K L$ SCALEPACK and DENZO (Otwinowski \& Minor, 1997); program(s) used to solve structure: DIRDIF99 (Beurskens et al., 1999); program(s) used to refine structure: SHELXL97 (Sheldrick, 1997); molecular graphics: ORTEP-3 for Windows (Farrugia, 1997); software used to prepare material for publication: WinGX (Farrugia, 1999).

The authors acknowledge funding from the EPSRC for this and related work.

\section{References}

Aldridge, S. \& Bresner, C. (2003). Coord. Chem. Rev. 244, 71-92. Aldridge, S., Bresner, C. \& Fallis, I. A. (2004). In preparation. Appel, A., Jäkle, F., Priermeier, T., Schmid, R. \& Wagner, M. (1996). Organometallics, 15, 1188-1194.

Bats, J. W., Ma, K. \& Wagner, M. (2002). Acta Cryst. C58, m129-m132.

Beurskens, P. T., Beurskens, G., de Gelder, R., García-Granda, S., Israel, R., Gould, R. O. \& Smits, J. M. M. (1999). The DIRDIF99 Program System. Technical Report of the Crystallography Laboratory, University of Nijmegen, The Netherlands.

Blessing, R. H. (1995). Acta Cryst. A51, 33-38.

Braga, D., Polito, M., Bracaccini, M., D'Addario, D., Tagliavini, E, Sturba, L. \& Grepioni, F. (2003). Organometallics, 22, 2142-2150.

Dusemund, C., Sandanayake, K. R. A. S. \& Shinkai, S. (1995). J. Chem. Soc. Chem. Commun. pp. 333-334.

Elschenbroich, C., Wolf, M., Pebler, J. \& Harms, K. (2004). Organometallics, 23, 454-459.

Farrugia, L. J. (1997). J. Appl. Cryst. 30, 565.

Farrugia, L. J. (1999). J. Appl. Cryst. 32, 837-838.

Hua, D. H., McGill, J. W., Ueda, M. \& Stephany, H. A. (2001). J. Organomet. Chem. 637-639, 832-836.

McVey, S., Morrison, I. G. \& Pauson, P. L. (1967). J. Chem. Soc. C, pp. $1847-$ 1850.

Nesmeyanov, A. N., Sazonowa, V. A. \& Drozd, V. N. (1959). Dokl. Akad. Nauk SSSR, 126, 1004-1008. (In Russian.)

Nonius (2000). COLLECT. Nonius BV, Delft, The Netherlands.

Ori, A. \& Shinkai, S. (1995). J. Chem. Soc. Chem. Commun. pp. 1771-1772.

Otwinowski, Z. \& Minor, W. (1997). Methods in Enzymology, Vol. 276,

Macromolecular Crystallography, Part A, edited by C. W. Carter Jr \& R. M.

Sweet, pp. 307-326. New York: Academic Press.

Rettig, S. J. \& Trotter, J. (1977). Can. J. Chem. 55, 3071-3075.

Shechter, H. \& Helling, J. F. (1961). J. Org. Chem. 26, 1034-1037.

Sheldrick, G. M. (1997). SHELXL97. University of Göttingen, Germany. 


\section{supporting information}

Acta Cryst. (2004). E60, m441-m443 [https://doi.org/10.1107/S1600536804006014]

Hydrogen-bonding motifs in the solid-state structure of ferroceneboronic acid

Christopher Bresner, Simon Aldridge, lan A. Fallis and Li-Ling Ooi

(I)

Crystal data

$\left[\mathrm{Fe}\left(\mathrm{C}_{5} \mathrm{H}_{5}\right)\left(\mathrm{C}_{5} \mathrm{H}_{6} \mathrm{BO}_{2}\right)\right]$

$F(000)=472$

$M_{r}=229.85$

Monoclinic, $P 2{ }_{1} / a$

$a=10.0680(7) \AA$

$b=7.0080(5) \AA$

$c=14.0300(13) \AA$

$\beta=106.320(3)^{\circ}$

$V=950.02(13) \AA^{3}$

$Z=4$

$D_{\mathrm{x}}=1.607 \mathrm{Mg} \mathrm{m}^{-3}$

Mo $K \alpha$ radiation, $\lambda=0.71073 \AA$

Cell parameters from 1453 reflections

$\theta=2.9-27.5^{\circ}$

$\mu=1.55 \mathrm{~mm}^{-1}$

$T=180 \mathrm{~K}$

Triangular plate, yellow

$0.13 \times 0.10 \times 0.03 \mathrm{~mm}$

Data collection

Nonius KappaCCD area-detector

2130 independent reflections

diffractometer

$\varphi$ scans

Absorption correction: multi-scan

(SORTAV; Blessing, 1995)

$T_{\min }=0.824, T_{\max }=0.955$

6820 measured reflections 1398 reflections with $I>2 \sigma(I)$

$R_{\text {int }}=0.092$

$\theta_{\max }=27.4^{\circ}, \theta_{\min }=3.0^{\circ}$

$h=-12 \rightarrow 12$

$k=-9 \rightarrow 9$

$l=-18 \rightarrow 18$

\section{Refinement}

Refinement on $F^{2}$

Least-squares matrix: full

$R\left[F^{2}>2 \sigma\left(F^{2}\right)\right]=0.055$

$w R\left(F^{2}\right)=0.105$

$S=1.03$

2130 reflections

129 parameters

0 restraints

$\mathrm{H}$-atom parameters constrained

$w=1 /\left[\sigma^{2}\left(F_{\mathrm{o}}^{2}\right)+(0.0264 P)^{2}+0.3652 P\right]$

where $P=\left(F_{\mathrm{o}}^{2}+2 F_{\mathrm{c}}^{2}\right) / 3$

$(\Delta / \sigma)_{\max }<0.001$

$\Delta \rho_{\max }=0.49$ e $\AA^{-3}$

$\Delta \rho_{\min }=-0.57$ e $\AA^{-3}$

Special details

Geometry. All e.s.d.'s (except the e.s.d. in the dihedral angle between two 1.s. planes) are estimated using the full covariance matrix. The cell e.s.d.'s are taken into account individually in the estimation of e.s.d.'s in distances, angles and torsion angles; correlations between e.s.d.'s in cell parameters are only used when they are defined by crystal symmetry. An approximate (isotropic) treatment of cell e.s.d.'s is used for estimating e.s.d.'s involving 1.s. planes.

Fractional atomic coordinates and isotropic or equivalent isotropic displacement parameters $\left(\AA^{2}\right)$

\begin{tabular}{lllll}
\hline & $x$ & $y$ & $z$ & $U_{\text {iso }} * U_{\text {eq }}$ \\
\hline Fe1 & $0.11105(5)$ & $0.00607(7)$ & $-0.22534(3)$ & $0.02057(18)$ \\
C1 & $0.0238(3)$ & $-0.0392(5)$ & $-0.3745(2)$ & $0.0178(8)$
\end{tabular}




$\begin{array}{lllll}\text { C2 } & -0.0747(4) & 0.0449(5) & -0.3306(2) & 0.0214(8) \\ \text { H2 } & -0.161 & -0.0095 & -0.3304 & 0.026^{*} \\ \text { C3 } & -0.0229(4) & 0.2212(5) & -0.2877(3) & 0.0248(9) \\ \text { H3 } & -0.0679 & 0.3048 & -0.2534 & 0.03^{*} \\ \text { C4 } & 0.1073(4) & 0.2525(5) & -0.3044(3) & 0.0255(9) \\ \text { H4 } & 0.1647 & 0.361 & -0.2838 & 0.031^{*} \\ \text { C5 } & 0.1378(4) & 0.0929(5) & -0.3577(2) & 0.0203(8) \\ \text { H5 } & 0.2191 & 0.0765 & -0.3785 & 0.024^{*} \\ \text { C6 } & 0.2554(6) & -0.1909(8) & -0.1623(3) & 0.0616(16) \\ \text { H6 } & 0.309 & -0.2606 & -0.1964 & 0.074^{*} \\ \text { C7 } & 0.1288(6) & -0.2463(6) & -0.1505(3) & 0.0519(13) \\ \text { H7 } & 0.0806 & -0.3607 & -0.1751 & 0.062^{*} \\ \text { C8 } & 0.0843(5) & -0.1061(6) & -0.0966(3) & 0.0388(11) \\ \text { H8 } & 0.0006 & -0.1085 & -0.078 & 0.047^{*} \\ \text { C9 } & 0.1839(4) & 0.0400(6) & -0.0743(2) & 0.0366(11) \\ \text { H9 } & 0.1795 & 0.1536 & -0.0384 & 0.044^{*} \\ \text { C10 } & 0.2912(4) & -0.0128(8) & -0.1148(3) & 0.0544(14) \\ \text { H10 } & 0.3728 & 0.0583 & -0.111 & 0.065^{*} \\ \text { B1 } & 0.0135(4) & -0.2396(6) & -0.4231(3) & 0.0163(9) \\ \text { O1 } & 0.1187(2) & -0.3230(3) & -0.45319(16) & 0.0202(6) \\ \text { H1 } & 0.185 & -0.2461 & -0.4448 & 0.03^{*} \\ \text { O2 } & -0.1077(2) & -0.3400(3) & -0.43684(17) & 0.0212(6) \\ \text { H2A } & -0.1029 & -0.4424 & -0.4667 & 0.032^{*} \\ & & & & \end{array}$

Atomic displacement parameters $\left(\AA^{2}\right)$

\begin{tabular}{lllllll}
\hline & $U^{11}$ & $U^{22}$ & $U^{33}$ & $U^{12}$ & $U^{13}$ & $U^{23}$ \\
\hline Fe1 & $0.0174(3)$ & $0.0271(3)$ & $0.0165(3)$ & $0.0004(3)$ & $0.0035(2)$ & $-0.0031(2)$ \\
C1 & $0.0145(18)$ & $0.0231(19)$ & $0.0138(16)$ & $0.0024(15)$ & $0.0007(14)$ & $0.0025(14)$ \\
C2 & $0.0137(18)$ & $0.025(2)$ & $0.0235(19)$ & $-0.0004(15)$ & $0.0014(15)$ & $-0.0024(15)$ \\
C3 & $0.022(2)$ & $0.0234(19)$ & $0.029(2)$ & $0.0057(17)$ & $0.0081(17)$ & $-0.0060(17)$ \\
C4 & $0.027(2)$ & $0.0185(19)$ & $0.031(2)$ & $-0.0040(17)$ & $0.0089(17)$ & $-0.0020(17)$ \\
C5 & $0.021(2)$ & $0.0210(19)$ & $0.0207(18)$ & $-0.0043(17)$ & $0.0087(15)$ & $-0.0028(16)$ \\
C6 & $0.067(4)$ & $0.091(4)$ & $0.019(2)$ & $0.054(3)$ & $0.000(2)$ & $0.003(3)$ \\
C7 & $0.082(4)$ & $0.040(3)$ & $0.027(2)$ & $0.007(3)$ & $0.004(3)$ & $0.007(2)$ \\
C8 & $0.042(3)$ & $0.057(3)$ & $0.016(2)$ & $-0.009(2)$ & $0.0051(19)$ & $0.002(2)$ \\
C9 & $0.044(3)$ & $0.046(3)$ & $0.0152(19)$ & $-0.010(2)$ & $0.0008(18)$ & $-0.0119(18)$ \\
C10 & $0.021(2)$ & $0.117(5)$ & $0.020(2)$ & $-0.004(3)$ & $-0.0025(17)$ & $0.016(3)$ \\
B1 & $0.010(2)$ & $0.023(2)$ & $0.0134(19)$ & $0.0051(17)$ & $-0.0002(15)$ & $0.0045(17)$ \\
O1 & $0.0139(13)$ & $0.0170(12)$ & $0.0296(14)$ & $-0.0025(10)$ & $0.0060(11)$ & $-0.0033(11)$ \\
O2 & $0.0183(14)$ & $0.0202(13)$ & $0.0263(14)$ & $0.0004(11)$ & $0.0081(11)$ & $-0.0056(11)$ \\
& & & & & & \\
\hline
\end{tabular}

Geometric parameters $\left(\AA,{ }^{o}\right)$

\begin{tabular}{llll}
\hline Fe1-C6 & $2.020(4)$ & $\mathrm{C} 4-\mathrm{C} 5$ & $1.426(5)$ \\
$\mathrm{Fe} 1-\mathrm{C} 10$ & $2.032(4)$ & $\mathrm{C} 4-\mathrm{H} 4$ & 0.95 \\
$\mathrm{Fe} 1-\mathrm{C} 7$ & $2.039(4)$ & $\mathrm{C} 5-\mathrm{H} 5$ & 0.95 \\
$\mathrm{Fe} 1-\mathrm{C} 5$ & $2.043(3)$ & $\mathrm{C} 6-\mathrm{C} 7$ & $1.387(7)$
\end{tabular}




\begin{tabular}{|c|c|c|c|}
\hline $\mathrm{Fe} 1-\mathrm{C} 4$ & $2.047(4)$ & $\mathrm{C} 6-\mathrm{C} 10$ & $1.413(7)$ \\
\hline $\mathrm{Fe} 1-\mathrm{C} 3$ & $2.048(3)$ & C6-H6 & 0.95 \\
\hline $\mathrm{Fe} 1-\mathrm{C} 2$ & $2.049(3)$ & $\mathrm{C} 7-\mathrm{C} 8$ & $1.388(6)$ \\
\hline $\mathrm{Fe} 1-\mathrm{C} 9$ & $2.053(3)$ & $\mathrm{C} 7-\mathrm{H} 7$ & 0.95 \\
\hline $\mathrm{Fe} 1-\mathrm{C} 1$ & $2.054(3)$ & $\mathrm{C} 8-\mathrm{C} 9$ & $1.406(6)$ \\
\hline $\mathrm{Fe} 1-\mathrm{C} 8$ & $2.056(4)$ & $\mathrm{C} 8-\mathrm{H} 8$ & 0.95 \\
\hline $\mathrm{C} 1-\mathrm{C} 2$ & $1.433(5)$ & $\mathrm{C} 9-\mathrm{C} 10$ & $1.404(6)$ \\
\hline $\mathrm{C} 1-\mathrm{C} 5$ & $1.441(5)$ & C9- $\mathrm{H} 9$ & 0.95 \\
\hline $\mathrm{C} 1-\mathrm{B} 1$ & $1.551(5)$ & $\mathrm{C} 10-\mathrm{H} 10$ & 0.95 \\
\hline $\mathrm{C} 2-\mathrm{C} 3$ & $1.408(5)$ & $\mathrm{B} 1-\mathrm{O} 2$ & $1.375(4)$ \\
\hline $\mathrm{C} 2-\mathrm{H} 2$ & 0.95 & $\mathrm{~B} 1-\mathrm{O} 1$ & $1.376(4)$ \\
\hline $\mathrm{C} 3-\mathrm{C} 4$ & $1.413(5)$ & $\mathrm{O} 1-\mathrm{H} 1$ & 0.84 \\
\hline $\mathrm{C} 3-\mathrm{H} 3$ & 0.95 & $\mathrm{O} 2-\mathrm{H} 2 \mathrm{~A}$ & 0.84 \\
\hline $\mathrm{C} 6-\mathrm{Fe} 1-\mathrm{C} 10$ & $40.8(2)$ & $\mathrm{C} 1-\mathrm{C} 2-\mathrm{H} 2$ & 125.4 \\
\hline $\mathrm{C} 6-\mathrm{Fe} 1-\mathrm{C} 7$ & $40.0(2)$ & $\mathrm{Fe} 1-\mathrm{C} 2-\mathrm{H} 2$ & 126.5 \\
\hline $\mathrm{C} 10-\mathrm{Fe} 1-\mathrm{C} 7$ & $67.7(2)$ & $\mathrm{C} 2-\mathrm{C} 3-\mathrm{C} 4$ & $108.3(3)$ \\
\hline $\mathrm{C} 6-\mathrm{Fe} 1-\mathrm{C} 5$ & $109.83(18)$ & $\mathrm{C} 2-\mathrm{C} 3-\mathrm{Fe} 1$ & $69.92(19)$ \\
\hline $\mathrm{C} 10-\mathrm{Fe} 1-\mathrm{C} 5$ & $113.32(16)$ & $\mathrm{C} 4-\mathrm{C} 3-\mathrm{Fe} 1$ & $69.8(2)$ \\
\hline $\mathrm{C} 7-\mathrm{Fe} 1-\mathrm{C} 5$ & $135.38(17)$ & $\mathrm{C} 2-\mathrm{C} 3-\mathrm{H} 3$ & 125.8 \\
\hline $\mathrm{C} 6-\mathrm{Fe} 1-\mathrm{C} 4$ & $135.7(2)$ & $\mathrm{C} 4-\mathrm{C} 3-\mathrm{H} 3$ & 125.8 \\
\hline $\mathrm{C} 10-\mathrm{Fe} 1-\mathrm{C} 4$ & $110.18(18)$ & $\mathrm{Fe} 1-\mathrm{C} 3-\mathrm{H} 3$ & 126 \\
\hline $\mathrm{C} 7-\mathrm{Fe} 1-\mathrm{C} 4$ & $175.16(19)$ & $\mathrm{C} 3-\mathrm{C} 4-\mathrm{C} 5$ & $108.2(3)$ \\
\hline $\mathrm{C} 5-\mathrm{Fe} 1-\mathrm{C} 4$ & $40.80(13)$ & $\mathrm{C} 3-\mathrm{C} 4-\mathrm{Fe} 1$ & $69.9(2)$ \\
\hline $\mathrm{C} 6-\mathrm{Fe} 1-\mathrm{C} 3$ & $175.5(2)$ & $\mathrm{C} 5-\mathrm{C} 4-\mathrm{Fe} 1$ & $69.4(2)$ \\
\hline $\mathrm{C} 10-\mathrm{Fe} 1-\mathrm{C} 3$ & $135.48(19)$ & $\mathrm{C} 3-\mathrm{C} 4-\mathrm{H} 4$ & 125.9 \\
\hline $\mathrm{C} 7-\mathrm{Fe} 1-\mathrm{C} 3$ & $144.1(2)$ & $\mathrm{C} 5-\mathrm{C} 4-\mathrm{H} 4$ & 125.9 \\
\hline $\mathrm{C} 5-\mathrm{Fe} 1-\mathrm{C} 3$ & $68.39(14)$ & $\mathrm{Fe} 1-\mathrm{C} 4-\mathrm{H} 4$ & 126.4 \\
\hline $\mathrm{C} 4-\mathrm{Fe} 1-\mathrm{C} 3$ & $40.36(14)$ & $\mathrm{C} 4-\mathrm{C} 5-\mathrm{C} 1$ & $108.1(3)$ \\
\hline $\mathrm{C} 6-\mathrm{Fe} 1-\mathrm{C} 2$ & $143.6(2)$ & $\mathrm{C} 4-\mathrm{C} 5-\mathrm{Fe} 1$ & 69.77 (19) \\
\hline $\mathrm{C} 10-\mathrm{Fe} 1-\mathrm{C} 2$ & $175.10(18)$ & $\mathrm{C} 1-\mathrm{C} 5-\mathrm{Fe} 1$ & 69.84 (19) \\
\hline $\mathrm{C} 7-\mathrm{Fe} 1-\mathrm{C} 2$ & $114.60(18)$ & $\mathrm{C} 4-\mathrm{C} 5-\mathrm{H} 5$ & 125.9 \\
\hline $\mathrm{C} 5-\mathrm{Fe} 1-\mathrm{C} 2$ & $68.41(14)$ & $\mathrm{C} 1-\mathrm{C} 5-\mathrm{H} 5$ & 125.9 \\
\hline $\mathrm{C} 4-\mathrm{Fe} 1-\mathrm{C} 2$ & $67.88(14)$ & $\mathrm{Fe} 1-\mathrm{C} 5-\mathrm{H} 5$ & 126 \\
\hline $\mathrm{C} 3-\mathrm{Fe} 1-\mathrm{C} 2$ & $40.21(13)$ & $\mathrm{C} 7-\mathrm{C} 6-\mathrm{C} 10$ & $108.2(4)$ \\
\hline $\mathrm{C} 6-\mathrm{Fe} 1-\mathrm{C} 9$ & $67.82(17)$ & $\mathrm{C} 7-\mathrm{C} 6-\mathrm{Fe} 1$ & $70.8(3)$ \\
\hline $\mathrm{C} 10-\mathrm{Fe} 1-\mathrm{C} 9$ & $40.20(17)$ & $\mathrm{C} 10-\mathrm{C} 6-\mathrm{Fe} 1$ & $70.1(3)$ \\
\hline $\mathrm{C} 7-\mathrm{Fe} 1-\mathrm{C} 9$ & $67.33(17)$ & $\mathrm{C} 7-\mathrm{C} 6-\mathrm{H} 6$ & 125.9 \\
\hline $\mathrm{C} 5-\mathrm{Fe} 1-\mathrm{C} 9$ & $143.53(16)$ & $\mathrm{C} 10-\mathrm{C} 6-\mathrm{H} 6$ & 125.9 \\
\hline $\mathrm{C} 4-\mathrm{Fe} 1-\mathrm{C} 9$ & $114.13(15)$ & $\mathrm{Fe} 1-\mathrm{C} 6-\mathrm{H} 6$ & 124.8 \\
\hline $\mathrm{C} 3-\mathrm{Fe} 1-\mathrm{C} 9$ & $110.92(15)$ & $\mathrm{C} 6-\mathrm{C} 7-\mathrm{C} 8$ & $108.3(4)$ \\
\hline $\mathrm{C} 2-\mathrm{Fe} 1-\mathrm{C} 9$ & $135.85(16)$ & $\mathrm{C} 6-\mathrm{C} 7-\mathrm{Fe} 1$ & $69.3(3)$ \\
\hline $\mathrm{C} 6-\mathrm{Fe} 1-\mathrm{C} 1$ & $112.94(16)$ & $\mathrm{C} 8-\mathrm{C} 7-\mathrm{Fe} 1$ & $70.8(2)$ \\
\hline $\mathrm{C} 10-\mathrm{Fe} 1-\mathrm{C} 1$ & $143.31(17)$ & $\mathrm{C} 6-\mathrm{C} 7-\mathrm{H} 7$ & 125.8 \\
\hline $\mathrm{C} 7-\mathrm{Fe} 1-\mathrm{C} 1$ & $109.94(15)$ & $\mathrm{C} 8-\mathrm{C} 7-\mathrm{H} 7$ & 125.8 \\
\hline $\mathrm{C} 5-\mathrm{Fe} 1-\mathrm{C} 1$ & $41.20(13)$ & $\mathrm{Fe} 1-\mathrm{C} 7-\mathrm{H} 7$ & 125.7 \\
\hline $\mathrm{C} 4-\mathrm{Fe} 1-\mathrm{C} 1$ & $68.94(13)$ & $\mathrm{C} 7-\mathrm{C} 8-\mathrm{C} 9$ & $108.6(4)$ \\
\hline $\mathrm{C} 3-\mathrm{Fe} 1-\mathrm{C} 1$ & $68.71(13)$ & $\mathrm{C} 7-\mathrm{C} 8-\mathrm{Fe} 1$ & $69.5(2)$ \\
\hline
\end{tabular}




\begin{tabular}{|c|c|c|c|}
\hline $\mathrm{C} 2-\mathrm{Fe} 1-\mathrm{C} 1$ & $40.89(13)$ & $\mathrm{C} 9-\mathrm{C} 8-\mathrm{Fe} 1$ & $69.9(2)$ \\
\hline $\mathrm{C} 9-\mathrm{Fe} 1-\mathrm{C} 1$ & $175.15(15)$ & $\mathrm{C} 7-\mathrm{C} 8-\mathrm{H} 8$ & 125.7 \\
\hline $\mathrm{C} 6-\mathrm{Fe} 1-\mathrm{C} 8$ & $67.02(19)$ & $\mathrm{C} 9-\mathrm{C} 8-\mathrm{H} 8$ & 125.7 \\
\hline $\mathrm{C} 10-\mathrm{Fe} 1-\mathrm{C} 8$ & $67.28(17)$ & $\mathrm{Fe} 1-\mathrm{C} 8-\mathrm{H} 8$ & 126.4 \\
\hline $\mathrm{C} 7-\mathrm{Fe} 1-\mathrm{C} 8$ & $39.64(17)$ & $\mathrm{C} 10-\mathrm{C} 9-\mathrm{C} 8$ & $107.4(4)$ \\
\hline $\mathrm{C} 5-\mathrm{Fe} 1-\mathrm{C} 8$ & $174.84(16)$ & $\mathrm{C} 10-\mathrm{C} 9-\mathrm{Fe} 1$ & $69.1(2)$ \\
\hline $\mathrm{C} 4-\mathrm{Fe} 1-\mathrm{C} 8$ & $144.27(16)$ & $\mathrm{C} 8-\mathrm{C} 9-\mathrm{Fe} 1$ & $70.1(2)$ \\
\hline $\mathrm{C} 3-\mathrm{Fe} 1-\mathrm{C} 8$ & $115.06(17)$ & $\mathrm{C} 10-\mathrm{C} 9-\mathrm{H} 9$ & 126.3 \\
\hline $\mathrm{C} 2-\mathrm{Fe} 1-\mathrm{C} 8$ & $111.44(16)$ & $\mathrm{C} 8-\mathrm{C} 9-\mathrm{H} 9$ & 126.3 \\
\hline $\mathrm{C} 9-\mathrm{Fe} 1-\mathrm{C} 8$ & $40.01(15)$ & $\mathrm{Fe} 1-\mathrm{C} 9-\mathrm{H} 9$ & 126 \\
\hline $\mathrm{C} 1-\mathrm{Fe} 1-\mathrm{C} 8$ & $135.39(15)$ & $\mathrm{C} 9-\mathrm{C} 10-\mathrm{C} 6$ & $107.5(4)$ \\
\hline $\mathrm{C} 2-\mathrm{C} 1-\mathrm{C} 5$ & $106.3(3)$ & $\mathrm{C} 9-\mathrm{C} 10-\mathrm{Fe} 1$ & $70.7(2)$ \\
\hline $\mathrm{C} 2-\mathrm{C} 1-\mathrm{B} 1$ & $126.3(3)$ & $\mathrm{C} 6-\mathrm{C} 10-\mathrm{Fe} 1$ & $69.1(2)$ \\
\hline $\mathrm{C} 5-\mathrm{C} 1-\mathrm{B} 1$ & $127.2(3)$ & $\mathrm{C} 9-\mathrm{C} 10-\mathrm{H} 10$ & 126.2 \\
\hline $\mathrm{C} 2-\mathrm{C} 1-\mathrm{Fe} 1$ & $69.35(18)$ & $\mathrm{C} 6-\mathrm{C} 10-\mathrm{H} 10$ & 126.2 \\
\hline $\mathrm{C} 5-\mathrm{C} 1-\mathrm{Fe} 1$ & $68.96(17)$ & $\mathrm{Fe} 1-\mathrm{C} 10-\mathrm{H} 10$ & 125.6 \\
\hline $\mathrm{B} 1-\mathrm{C} 1-\mathrm{Fe} 1$ & $123.0(2)$ & $\mathrm{O} 2-\mathrm{B} 1-\mathrm{O} 1$ & $118.0(3)$ \\
\hline $\mathrm{C} 3-\mathrm{C} 2-\mathrm{C} 1$ & $109.1(3)$ & $\mathrm{O} 2-\mathrm{B} 1-\mathrm{C} 1$ & $118.1(3)$ \\
\hline $\mathrm{C} 3-\mathrm{C} 2-\mathrm{Fe} 1$ & $69.86(19)$ & $\mathrm{O} 1-\mathrm{B} 1-\mathrm{C} 1$ & $123.9(3)$ \\
\hline $\mathrm{C} 1-\mathrm{C} 2-\mathrm{Fe} 1$ & $69.76(18)$ & $\mathrm{B} 1-\mathrm{O} 1-\mathrm{H} 1$ & 109.5 \\
\hline $\mathrm{C} 3-\mathrm{C} 2-\mathrm{H} 2$ & 125.5 & $\mathrm{~B} 1-\mathrm{O} 2-\mathrm{H} 2 \mathrm{~A}$ & 109.5 \\
\hline $\mathrm{C} 6-\mathrm{Fe} 1-\mathrm{C} 1-\mathrm{C} 2$ & $147.9(3)$ & $\mathrm{C} 3-\mathrm{Fe} 1-\mathrm{C} 5-\mathrm{C} 1$ & $-81.9(2)$ \\
\hline $\mathrm{C} 10-\mathrm{Fe} 1-\mathrm{C} 1-\mathrm{C} 2$ & $-176.0(3)$ & $\mathrm{C} 2-\mathrm{Fe} 1-\mathrm{C} 5-\mathrm{C} 1$ & $-38.53(19)$ \\
\hline $\mathrm{C} 7-\mathrm{Fe} 1-\mathrm{C} 1-\mathrm{C} 2$ & $104.9(3)$ & $\mathrm{C} 9-\mathrm{Fe} 1-\mathrm{C} 5-\mathrm{C} 1$ & $-178.3(2)$ \\
\hline $\mathrm{C} 5-\mathrm{Fe} 1-\mathrm{C} 1-\mathrm{C} 2$ & $-117.8(3)$ & $\mathrm{C} 10-\mathrm{Fe} 1-\mathrm{C} 6-\mathrm{C} 7$ & $118.5(4)$ \\
\hline $\mathrm{C} 4-\mathrm{Fe} 1-\mathrm{C} 1-\mathrm{C} 2$ & $-80.1(2)$ & $\mathrm{C} 5-\mathrm{Fe} 1-\mathrm{C} 6-\mathrm{C} 7$ & $-138.4(3)$ \\
\hline $\mathrm{C} 3-\mathrm{Fe} 1-\mathrm{C} 1-\mathrm{C} 2$ & $-36.7(2)$ & $\mathrm{C} 4-\mathrm{Fe} 1-\mathrm{C} 6-\mathrm{C} 7$ & $-176.9(2)$ \\
\hline $\mathrm{C} 8-\mathrm{Fe} 1-\mathrm{C} 1-\mathrm{C} 2$ & $67.9(3)$ & $\mathrm{C} 2-\mathrm{Fe} 1-\mathrm{C} 6-\mathrm{C} 7$ & $-58.2(4)$ \\
\hline $\mathrm{C} 6-\mathrm{Fe} 1-\mathrm{C} 1-\mathrm{C} 5$ & $-94.4(3)$ & $\mathrm{C} 9-\mathrm{Fe} 1-\mathrm{C} 6-\mathrm{C} 7$ & $80.7(3)$ \\
\hline $\mathrm{C} 10-\mathrm{Fe} 1-\mathrm{C} 1-\mathrm{C} 5$ & $-58.2(4)$ & $\mathrm{C} 1-\mathrm{Fe} 1-\mathrm{C} 6-\mathrm{C} 7$ & $-94.1(3)$ \\
\hline $\mathrm{C} 7-\mathrm{Fe} 1-\mathrm{C} 1-\mathrm{C} 5$ & $-137.3(2)$ & $\mathrm{C} 8-\mathrm{Fe} 1-\mathrm{C} 6-\mathrm{C} 7$ & $37.2(3)$ \\
\hline $\mathrm{C} 4-\mathrm{Fe} 1-\mathrm{C} 1-\mathrm{C} 5$ & $37.7(2)$ & $\mathrm{C} 7-\mathrm{Fe} 1-\mathrm{C} 6-\mathrm{C} 10$ & $-118.5(4)$ \\
\hline $\mathrm{C} 3-\mathrm{Fe} 1-\mathrm{C} 1-\mathrm{C} 5$ & $81.1(2)$ & $\mathrm{C} 5-\mathrm{Fe} 1-\mathrm{C} 6-\mathrm{C} 10$ & $103.1(3)$ \\
\hline $\mathrm{C} 2-\mathrm{Fe} 1-\mathrm{C} 1-\mathrm{C} 5$ & $117.8(3)$ & $\mathrm{C} 4-\mathrm{Fe} 1-\mathrm{C} 6-\mathrm{C} 10$ & $64.6(3)$ \\
\hline $\mathrm{C} 8-\mathrm{Fe} 1-\mathrm{C} 1-\mathrm{C} 5$ & $-174.3(2)$ & $\mathrm{C} 2-\mathrm{Fe} 1-\mathrm{C} 6-\mathrm{C} 10$ & $-176.7(3)$ \\
\hline $\mathrm{C} 6-\mathrm{Fe} 1-\mathrm{C} 1-\mathrm{B} 1$ & $27.2(4)$ & $\mathrm{C} 9-\mathrm{Fe} 1-\mathrm{C} 6-\mathrm{C} 10$ & $-37.8(3)$ \\
\hline $\mathrm{C} 10-\mathrm{Fe} 1-\mathrm{C} 1-\mathrm{B} 1$ & $63.3(4)$ & $\mathrm{C} 1-\mathrm{Fe} 1-\mathrm{C} 6-\mathrm{C} 10$ & $147.4(3)$ \\
\hline $\mathrm{C} 7-\mathrm{Fe} 1-\mathrm{C} 1-\mathrm{B} 1$ & $-15.8(4)$ & $\mathrm{C} 8-\mathrm{Fe} 1-\mathrm{C} 6-\mathrm{C} 10$ & $-81.3(3)$ \\
\hline $\mathrm{C} 5-\mathrm{Fe} 1-\mathrm{C} 1-\mathrm{B} 1$ & $121.5(4)$ & $\mathrm{C} 10-\mathrm{C} 6-\mathrm{C} 7-\mathrm{C} 8$ & $0.1(5)$ \\
\hline $\mathrm{C} 4-\mathrm{Fe} 1-\mathrm{C} 1-\mathrm{B} 1$ & $159.2(3)$ & $\mathrm{Fe} 1-\mathrm{C} 6-\mathrm{C} 7-\mathrm{C} 8$ & $-60.3(3)$ \\
\hline $\mathrm{C} 3-\mathrm{Fe} 1-\mathrm{C} 1-\mathrm{B} 1$ & $-157.4(3)$ & $\mathrm{C} 10-\mathrm{C} 6-\mathrm{C} 7-\mathrm{Fe} 1$ & $60.4(3)$ \\
\hline $\mathrm{C} 2-\mathrm{Fe} 1-\mathrm{C} 1-\mathrm{B} 1$ & $-120.7(4)$ & $\mathrm{C} 10-\mathrm{Fe} 1-\mathrm{C} 7-\mathrm{C} 6$ & $-38.4(3)$ \\
\hline $\mathrm{C} 8-\mathrm{Fe} 1-\mathrm{C} 1-\mathrm{B} 1$ & $-52.8(4)$ & $\mathrm{C} 5-\mathrm{Fe} 1-\mathrm{C} 7-\mathrm{C} 6$ & $62.8(4)$ \\
\hline $\mathrm{C} 5-\mathrm{C} 1-\mathrm{C} 2-\mathrm{C} 3$ & $-0.4(4)$ & $\mathrm{C} 3-\mathrm{Fe} 1-\mathrm{C} 7-\mathrm{C} 6$ & $-176.8(3)$ \\
\hline $\mathrm{B} 1-\mathrm{C} 1-\mathrm{C} 2-\mathrm{C} 3$ & $175.4(3)$ & $\mathrm{C} 2-\mathrm{Fe} 1-\mathrm{C} 7-\mathrm{C} 6$ & $146.4(3)$ \\
\hline $\mathrm{Fe} 1-\mathrm{C} 1-\mathrm{C} 2-\mathrm{C} 3$ & $58.9(2)$ & $\mathrm{C} 9-\mathrm{Fe} 1-\mathrm{C} 7-\mathrm{C} 6$ & $-82.0(3)$ \\
\hline $\mathrm{C} 5-\mathrm{C} 1-\mathrm{C} 2-\mathrm{Fe} 1$ & $-59.4(2)$ & $\mathrm{C} 1-\mathrm{Fe} 1-\mathrm{C} 7-\mathrm{C} 6$ & $102.3(3)$ \\
\hline
\end{tabular}




\begin{tabular}{|c|c|c|c|}
\hline $\mathrm{B} 1-\mathrm{C} 1-\mathrm{C} 2-\mathrm{Fe} 1$ & $116.4(3)$ & $\mathrm{C} 8-\mathrm{Fe} 1-\mathrm{C} 7-\mathrm{C} 6$ & $-119.2(4)$ \\
\hline $\mathrm{C} 6-\mathrm{Fe} 1-\mathrm{C} 2-\mathrm{C} 3$ & $-176.1(3)$ & $\mathrm{C} 6-\mathrm{Fe} 1-\mathrm{C} 7-\mathrm{C} 8$ & $119.2(4)$ \\
\hline $\mathrm{C} 7-\mathrm{Fe} 1-\mathrm{C} 2-\mathrm{C} 3$ & $147.0(2)$ & $\mathrm{C} 10-\mathrm{Fe} 1-\mathrm{C} 7-\mathrm{C} 8$ & $80.8(3)$ \\
\hline $\mathrm{C} 5-\mathrm{Fe} 1-\mathrm{C} 2-\mathrm{C} 3$ & $-81.6(2)$ & $\mathrm{C} 5-\mathrm{Fe} 1-\mathrm{C} 7-\mathrm{C} 8$ & $-178.0(2)$ \\
\hline $\mathrm{C} 4-\mathrm{Fe} 1-\mathrm{C} 2-\mathrm{C} 3$ & $-37.5(2)$ & $\mathrm{C} 3-\mathrm{Fe} 1-\mathrm{C} 7-\mathrm{C} 8$ & $-57.6(4)$ \\
\hline $\mathrm{C} 9-\mathrm{Fe} 1-\mathrm{C} 2-\mathrm{C} 3$ & $64.9(3)$ & $\mathrm{C} 2-\mathrm{Fe} 1-\mathrm{C} 7-\mathrm{C} 8$ & $-94.4(3)$ \\
\hline $\mathrm{C} 1-\mathrm{Fe} 1-\mathrm{C} 2-\mathrm{C} 3$ & $-120.4(3)$ & $\mathrm{C} 9-\mathrm{Fe} 1-\mathrm{C} 7-\mathrm{C} 8$ & $37.2(3)$ \\
\hline $\mathrm{C} 8-\mathrm{Fe} 1-\mathrm{C} 2-\mathrm{C} 3$ & $103.9(2)$ & $\mathrm{C} 1-\mathrm{Fe} 1-\mathrm{C} 7-\mathrm{C} 8$ & $-138.5(3)$ \\
\hline $\mathrm{C} 6-\mathrm{Fe} 1-\mathrm{C} 2-\mathrm{C} 1$ & $-55.7(4)$ & $\mathrm{C} 6-\mathrm{C} 7-\mathrm{C} 8-\mathrm{C} 9$ & $0.2(5)$ \\
\hline $\mathrm{C} 7-\mathrm{Fe} 1-\mathrm{C} 2-\mathrm{C} 1$ & $-92.5(2)$ & $\mathrm{Fe} 1-\mathrm{C} 7-\mathrm{C} 8-\mathrm{C} 9$ & $-59.2(3)$ \\
\hline $\mathrm{C} 5-\mathrm{Fe} 1-\mathrm{C} 2-\mathrm{C} 1$ & $38.81(19)$ & $\mathrm{C} 6-\mathrm{C} 7-\mathrm{C} 8-\mathrm{Fe} 1$ & $59.3(3)$ \\
\hline $\mathrm{C} 4-\mathrm{Fe} 1-\mathrm{C} 2-\mathrm{C} 1$ & $82.9(2)$ & $\mathrm{C} 6-\mathrm{Fe} 1-\mathrm{C} 8-\mathrm{C} 7$ & $-37.5(3)$ \\
\hline $\mathrm{C} 3-\mathrm{Fe} 1-\mathrm{C} 2-\mathrm{C} 1$ & $120.4(3)$ & $\mathrm{C} 10-\mathrm{Fe} 1-\mathrm{C} 8-\mathrm{C} 7$ & $-82.0(3)$ \\
\hline $\mathrm{C} 9-\mathrm{Fe} 1-\mathrm{C} 2-\mathrm{C} 1$ & $-174.7(2)$ & $\mathrm{C} 4-\mathrm{Fe} 1-\mathrm{C} 8-\mathrm{C} 7$ & $-175.3(3)$ \\
\hline $\mathrm{C} 8-\mathrm{Fe} 1-\mathrm{C} 2-\mathrm{C} 1$ & $-135.6(2)$ & $\mathrm{C} 3-\mathrm{Fe} 1-\mathrm{C} 8-\mathrm{C} 7$ & $146.9(3)$ \\
\hline $\mathrm{C} 1-\mathrm{C} 2-\mathrm{C} 3-\mathrm{C} 4$ & $0.6(4)$ & $\mathrm{C} 2-\mathrm{Fe} 1-\mathrm{C} 8-\mathrm{C} 7$ & $103.1(3)$ \\
\hline $\mathrm{Fe} 1-\mathrm{C} 2-\mathrm{C} 3-\mathrm{C} 4$ & $59.4(2)$ & $\mathrm{C} 9-\mathrm{Fe} 1-\mathrm{C} 8-\mathrm{C} 7$ & $-119.9(4)$ \\
\hline $\mathrm{C} 1-\mathrm{C} 2-\mathrm{C} 3-\mathrm{Fe} 1$ & $-58.9(2)$ & $\mathrm{C} 1-\mathrm{Fe} 1-\mathrm{C} 8-\mathrm{C} 7$ & $62.4(4)$ \\
\hline $\mathrm{C} 10-\mathrm{Fe} 1-\mathrm{C} 3-\mathrm{C} 2$ & $-176.5(2)$ & $\mathrm{C} 6-\mathrm{Fe} 1-\mathrm{C} 8-\mathrm{C} 9$ & $82.4(3)$ \\
\hline $\mathrm{C} 7-\mathrm{Fe} 1-\mathrm{C} 3-\mathrm{C} 2$ & $-57.6(3)$ & $\mathrm{C} 10-\mathrm{Fe} 1-\mathrm{C} 8-\mathrm{C} 9$ & $37.9(3)$ \\
\hline $\mathrm{C} 5-\mathrm{Fe} 1-\mathrm{C} 3-\mathrm{C} 2$ & $81.7(2)$ & $\mathrm{C} 7-\mathrm{Fe} 1-\mathrm{C} 8-\mathrm{C} 9$ & $119.9(4)$ \\
\hline $\mathrm{C} 4-\mathrm{Fe} 1-\mathrm{C} 3-\mathrm{C} 2$ & $119.4(3)$ & $\mathrm{C} 4-\mathrm{Fe} 1-\mathrm{C} 8-\mathrm{C} 9$ & $-55.4(4)$ \\
\hline $\mathrm{C} 9-\mathrm{Fe} 1-\mathrm{C} 3-\mathrm{C} 2$ & $-137.5(2)$ & $\mathrm{C} 3-\mathrm{Fe} 1-\mathrm{C} 8-\mathrm{C} 9$ & $-93.2(3)$ \\
\hline $\mathrm{C} 1-\mathrm{Fe} 1-\mathrm{C} 3-\mathrm{C} 2$ & $37.3(2)$ & $\mathrm{C} 2-\mathrm{Fe} 1-\mathrm{C} 8-\mathrm{C} 9$ & $-137.0(3)$ \\
\hline $\mathrm{C} 8-\mathrm{Fe} 1-\mathrm{C} 3-\mathrm{C} 2$ & $-94.1(2)$ & $\mathrm{C} 1-\mathrm{Fe} 1-\mathrm{C} 8-\mathrm{C} 9$ & $-177.7(2)$ \\
\hline $\mathrm{C} 10-\mathrm{Fe} 1-\mathrm{C} 3-\mathrm{C} 4$ & $64.1(3)$ & $\mathrm{C} 7-\mathrm{C} 8-\mathrm{C} 9-\mathrm{C} 10$ & $-0.3(4)$ \\
\hline $\mathrm{C} 7-\mathrm{Fe} 1-\mathrm{C} 3-\mathrm{C} 4$ & $-177.0(3)$ & $\mathrm{Fe} 1-\mathrm{C} 8-\mathrm{C} 9-\mathrm{C} 10$ & $-59.3(3)$ \\
\hline $\mathrm{C} 5-\mathrm{Fe} 1-\mathrm{C} 3-\mathrm{C} 4$ & $-37.7(2)$ & $\mathrm{C} 7-\mathrm{C} 8-\mathrm{C} 9-\mathrm{Fe} 1$ & $59.0(3)$ \\
\hline $\mathrm{C} 2-\mathrm{Fe} 1-\mathrm{C} 3-\mathrm{C} 4$ & $-119.4(3)$ & $\mathrm{C} 6-\mathrm{Fe} 1-\mathrm{C} 9-\mathrm{C} 10$ & $38.4(3)$ \\
\hline $\mathrm{C} 9-\mathrm{Fe} 1-\mathrm{C} 3-\mathrm{C} 4$ & $103.1(2)$ & $\mathrm{C} 7-\mathrm{Fe} 1-\mathrm{C} 9-\mathrm{C} 10$ & $81.8(3)$ \\
\hline $\mathrm{C} 1-\mathrm{Fe} 1-\mathrm{C} 3-\mathrm{C} 4$ & $-82.1(2)$ & $\mathrm{C} 5-\mathrm{Fe} 1-\mathrm{C} 9-\mathrm{C} 10$ & $-55.3(4)$ \\
\hline $\mathrm{C} 8-\mathrm{Fe} 1-\mathrm{C} 3-\mathrm{C} 4$ & $146.5(2)$ & $\mathrm{C} 4-\mathrm{Fe} 1-\mathrm{C} 9-\mathrm{C} 10$ & $-93.2(3)$ \\
\hline $\mathrm{C} 2-\mathrm{C} 3-\mathrm{C} 4-\mathrm{C} 5$ & $-0.5(4)$ & $\mathrm{C} 3-\mathrm{Fe} 1-\mathrm{C} 9-\mathrm{C} 10$ & $-136.9(3)$ \\
\hline $\mathrm{Fe} 1-\mathrm{C} 3-\mathrm{C} 4-\mathrm{C} 5$ & $59.0(2)$ & $\mathrm{C} 2-\mathrm{Fe} 1-\mathrm{C} 9-\mathrm{C} 10$ & $-175.7(3)$ \\
\hline $\mathrm{C} 2-\mathrm{C} 3-\mathrm{C} 4-\mathrm{Fe} 1$ & $-59.5(2)$ & $\mathrm{C} 8-\mathrm{Fe} 1-\mathrm{C} 9-\mathrm{C} 10$ & $118.6(4)$ \\
\hline $\mathrm{C} 6-\mathrm{Fe} 1-\mathrm{C} 4-\mathrm{C} 3$ & $-176.8(2)$ & $\mathrm{C} 6-\mathrm{Fe} 1-\mathrm{C} 9-\mathrm{C} 8$ & $-80.2(3)$ \\
\hline $\mathrm{C} 10-\mathrm{Fe} 1-\mathrm{C} 4-\mathrm{C} 3$ & $-137.8(2)$ & $\mathrm{C} 10-\mathrm{Fe} 1-\mathrm{C} 9-\mathrm{C} 8$ & $-118.6(4)$ \\
\hline $\mathrm{C} 5-\mathrm{Fe} 1-\mathrm{C} 4-\mathrm{C} 3$ & $119.5(3)$ & $\mathrm{C} 7-\mathrm{Fe} 1-\mathrm{C} 9-\mathrm{C} 8$ & $-36.8(3)$ \\
\hline $\mathrm{C} 2-\mathrm{Fe} 1-\mathrm{C} 4-\mathrm{C} 3$ & $37.4(2)$ & $\mathrm{C} 5-\mathrm{Fe} 1-\mathrm{C} 9-\mathrm{C} 8$ & $-173.9(3)$ \\
\hline $\mathrm{C} 9-\mathrm{Fe} 1-\mathrm{C} 4-\mathrm{C} 3$ & $-94.4(2)$ & $\mathrm{C} 4-\mathrm{Fe} 1-\mathrm{C} 9-\mathrm{C} 8$ & $148.2(3)$ \\
\hline $\mathrm{C} 1-\mathrm{Fe} 1-\mathrm{C} 4-\mathrm{C} 3$ & $81.5(2)$ & $\mathrm{C} 3-\mathrm{Fe} 1-\mathrm{C} 9-\mathrm{C} 8$ & $104.5(3)$ \\
\hline $\mathrm{C} 8-\mathrm{Fe} 1-\mathrm{C} 4-\mathrm{C} 3$ & $-59.0(3)$ & $\mathrm{C} 2-\mathrm{Fe} 1-\mathrm{C} 9-\mathrm{C} 8$ & $65.7(3)$ \\
\hline $\mathrm{C} 6-\mathrm{Fe} 1-\mathrm{C} 4-\mathrm{C} 5$ & $63.7(3)$ & $\mathrm{C} 8-\mathrm{C} 9-\mathrm{C} 10-\mathrm{C} 6$ & $0.4(4)$ \\
\hline $\mathrm{C} 10-\mathrm{Fe} 1-\mathrm{C} 4-\mathrm{C} 5$ & $102.7(2)$ & $\mathrm{Fe} 1-\mathrm{C} 9-\mathrm{C} 10-\mathrm{C} 6$ & $-59.5(3)$ \\
\hline $\mathrm{C} 3-\mathrm{Fe} 1-\mathrm{C} 4-\mathrm{C} 5$ & $-119.5(3)$ & $\mathrm{C} 8-\mathrm{C} 9-\mathrm{C} 10-\mathrm{Fe} 1$ & $59.9(3)$ \\
\hline $\mathrm{C} 2-\mathrm{Fe} 1-\mathrm{C} 4-\mathrm{C} 5$ & $-82.1(2)$ & $\mathrm{C} 7-\mathrm{C} 6-\mathrm{C} 10-\mathrm{C} 9$ & $-0.3(5)$ \\
\hline $\mathrm{C} 9-\mathrm{Fe} 1-\mathrm{C} 4-\mathrm{C} 5$ & $146.1(2)$ & $\mathrm{Fe} 1-\mathrm{C} 6-\mathrm{C} 10-\mathrm{C} 9$ & $60.5(3)$ \\
\hline $\mathrm{C} 1-\mathrm{Fe} 1-\mathrm{C} 4-\mathrm{C} 5$ & $-38.0(2)$ & $\mathrm{C} 7-\mathrm{C} 6-\mathrm{C} 10-\mathrm{Fe} 1$ & $-60.8(3)$ \\
\hline
\end{tabular}




$\begin{array}{llll}\mathrm{C} 8-\mathrm{Fe} 1-\mathrm{C} 4-\mathrm{C} 5 & -178.5(3) & \mathrm{C} 6-\mathrm{Fe} 1-\mathrm{C} 10-\mathrm{C} 9 & -118.4(4) \\ \mathrm{C} 3-\mathrm{C} 4-\mathrm{C} 5-\mathrm{C} 1 & 0.2(4) & \mathrm{C} 7-\mathrm{Fe} 1-\mathrm{C} 10-\mathrm{C} 9 & -80.8(3) \\ \mathrm{Fe} 1-\mathrm{C} 4-\mathrm{C} 5-\mathrm{C} 1 & 59.5(2) & \mathrm{C} 5-\mathrm{Fe} 1-\mathrm{C} 10-\mathrm{C} 9 & 147.8(2) \\ \mathrm{C} 3-\mathrm{C} 4-\mathrm{C} 5-\mathrm{Fe} 1 & -59.3(2) & \mathrm{C} 4-\mathrm{Fe} 1-\mathrm{C} 10-\mathrm{C} 9 & 103.9(3) \\ \mathrm{C} 2-\mathrm{C} 1-\mathrm{C} 5-\mathrm{C} 4 & 0.1(4) & \mathrm{C} 3-\mathrm{Fe} 1-\mathrm{C} 10-\mathrm{C} 9 & 65.5(3) \\ \mathrm{B} 1-\mathrm{C} 1-\mathrm{C} 5-\mathrm{C} 4 & -175.6(3) & \mathrm{C} 1-\mathrm{Fe} 1-\mathrm{C} 10-\mathrm{C} 9 & -174.6(2) \\ \mathrm{Fe} 1-\mathrm{C} 1-\mathrm{C} 5-\mathrm{C} 4 & -59.5(2) & \mathrm{C} 8-\mathrm{Fe} 1-\mathrm{C} 10-\mathrm{C} 9 & -37.7(3) \\ \mathrm{C} 2-\mathrm{C} 1-\mathrm{C} 5-\mathrm{Fe} 1 & 59.6(2) & \mathrm{C} 7-\mathrm{Fe} 1-\mathrm{C} 10-\mathrm{C} 6 & 37.6(3) \\ \mathrm{B} 1-\mathrm{C} 1-\mathrm{C} 5-\mathrm{Fe} 1 & -116.1(3) & \mathrm{C} 5-\mathrm{Fe} 1-\mathrm{C} 10-\mathrm{C} 6 & -93.8(3) \\ \mathrm{C} 6-\mathrm{Fe} 1-\mathrm{C} 5-\mathrm{C} 4 & -138.2(3) & \mathrm{C} 4-\mathrm{Fe} 1-\mathrm{C} 10-\mathrm{C} 6 & -137.8(3) \\ \mathrm{C} 10-\mathrm{Fe} 1-\mathrm{C} 5-\mathrm{C} 4 & -94.3(3) & \mathrm{C} 3-\mathrm{Fe} 1-\mathrm{C} 10-\mathrm{C} 6 & -176.1(3) \\ \mathrm{C} 7-\mathrm{Fe} 1-\mathrm{C} 5-\mathrm{C} 4 & -175.6(3) & \mathrm{C} 9-\mathrm{Fe} 1-\mathrm{C} 10-\mathrm{C} 6 & 118.4(4) \\ \mathrm{C} 3-\mathrm{Fe} 1-\mathrm{C} 5-\mathrm{C} 4 & 37.3(2) & \mathrm{C} 1-\mathrm{Fe} 1-\mathrm{C} 10-\mathrm{C} 6 & -56.2(4) \\ \mathrm{C} 2-\mathrm{Fe} 1-\mathrm{C} 5-\mathrm{C} 4 & 80.7(2) & \mathrm{C} 8-\mathrm{Fe} 1-\mathrm{C} 10-\mathrm{C} 6 & 80.6(3) \\ \mathrm{C} 9-\mathrm{Fe} 1-\mathrm{C} 5-\mathrm{C} 4 & -59.0(3) & \mathrm{C} 2-\mathrm{C} 1-\mathrm{B} 1-\mathrm{O} 2 & 8.3(5) \\ \mathrm{C} 1-\mathrm{Fe} 1-\mathrm{C} 5-\mathrm{C} 4 & 119.2(3) & \mathrm{C} 5-\mathrm{C} 1-\mathrm{B} 1-\mathrm{O} 2 & -176.8(3) \\ \mathrm{C} 6-\mathrm{Fe} 1-\mathrm{C} 5-\mathrm{C} 1 & 102.5(3) & \mathrm{Fe} 1-\mathrm{C} 1-\mathrm{B} 1-\mathrm{O} 2 & 95.7(3) \\ \mathrm{C} 10-\mathrm{Fe} 1-\mathrm{C} 5-\mathrm{C} 1 & 146.4(2) & \mathrm{C} 2-\mathrm{C} 1-\mathrm{B} 1-\mathrm{O} 1 & -171.6(3) \\ \mathrm{C} 7-\mathrm{Fe} 1-\mathrm{C} 5-\mathrm{C} 1 & 65.1(3) & \mathrm{C} 5-\mathrm{C} 1-\mathrm{B} 1-\mathrm{O} 1 & 3.3(5) \\ \mathrm{C} 4-\mathrm{Fe} 1-\mathrm{C} 5-\mathrm{C} 1 & -119.2(3) & \mathrm{Fe} 1-\mathrm{C} 1-\mathrm{B} 1-\mathrm{O} 1 & -84.1(4)\end{array}$

Hydrogen-bond geometry $\left(A,{ }^{\circ}\right)$

\begin{tabular}{lllll}
\hline$D-\mathrm{H} \cdots A$ & $D-\mathrm{H}$ & $\mathrm{H} \cdots A$ & $D \cdots A$ & $D-\mathrm{H} \cdots A$ \\
\hline $\mathrm{O} 1-\mathrm{H} 1 \cdots \mathrm{O} 2^{\mathrm{i}}$ & 0.84 & 2.14 & $2.930(3)$ & 156 \\
$\mathrm{O} 2-\mathrm{H} 2 A \cdots \mathrm{O} 1^{\mathrm{ii}}$ & 0.84 & 1.97 & $2.806(3)$ & 172 \\
\hline
\end{tabular}

Symmetry codes: (i) $x+1 / 2,-y-1 / 2, z$; (ii) $-x,-y-1,-z-1$. 